\title{
Significados que las gestantes hospitalizadas le atribuyen a la experiencia de tener preeclampsia ${ }^{1}$
}

\author{
Norma Noguera Ortiz² \\ Lucy Muñoz de Rodríguez³
}

doi:10.11144/Javeriana.IE16-1.sgha

Cómo citar: Noguera Ortiz N, Muñoz de Rodríguez L. Significados que las gestantes hospitalizadas le atribuyen a la experiencia de tener preeclampsia. Investig. Enferm. Imagen Desarro. 2014;16(1):27-48. doi:10.11144/Javeriana.IE16-1.sgha

1. Artículo de investigación. Recibido: 01 de octubre de 2013. Revisado: 05 de febrero de 2014. Aprobado: 10 de febrero de 2014.

2. Enfermera, especialista en Enfermeria Materno Perinatal con énfasis Familiar, magíster en Enfermeria. Profesora asistente, Departamento de Enfermería Clínica, Facultad de Enfermería, Pontificia Universidad Javeriana, Bogotá, Colombia. Correo electrónico: nogueran @javeriana.edu.co

3. Enfermera, especialista en Enfermería Obstétrica, magíster en Enfermería con énfasis en Salud Familiar. Profesora pensionada, Facultad de Enfermería, Universidad Nacional de Colombia. Correo electrónico: 1munozdr@unal.edu.co 


\section{Resumen}

Este estudio tuvo como objetivo describir los significados que un grupo de gestantes hospitalizadas le atribuyen a la experiencia de tener preeclampsia. Es una investigación cualitativa tipo etnoenfermería. Para la recolección de la información se tuvo en cuenta la Guía observación-participación-reflexión, propuesta por Leininger. Se hicieron entrevistas abiertas a profundidad a ocho gestantes como informantes clave y a cinco enfermeras como informantes generales. La muestra se obtuvo por saturación de la información. En el análisis de datos se aplicaron las cuatro fases propuestas por Leininger: recolección de la información, identificación y categorización de los descriptores y componentes, análisis contextual y de patrones y establecimiento de unos temas centrales. Los resultados de esta investigación aportan conocimiento teórico para brindar cuidado culturalmente congruente a gestantes con preeclampsia y, así, ser un complemento para su atención integral. Las mujeres requieren que el equipo de salud que las atiende les informen de manera clara y completa sobre su situación de salud, la de su hijo por nacer y los cuidados que necesitan. Las mujeres conocen sobre signos y síntomas de alarma para la preeclampsia, pero no los perciben. Los cambios emocionales, sociales, espirituales, producidos en la mujer, por su enfermedad y por la hospitalización, necesitan ser tenidos en cuenta para el cuidado de enfermería.

Palabras clave: preeclampsia, mujeres embarazadas, investigación cualitativa, enfermeria, enfermería transcultural.

Palabras clave descriptor: Preeclampsia, mujeres embarazadas, investigación cualitativa, enfermería, enfermería transcultural.

\section{Meanings hospitalized pregnant attribute to the experience of having preeclampsia}

\section{Abstract}

This study aimed at describing the meanings that a group of hospitalized pregnant attributed to the experience of having preeclampsia. It is a qualitative research of ethno nursing type. To collect the information the Observation-participation-reflection guide, given by Leininger was taken into account. Open interviews were conducted to eight pregnant women as key informants and five nurses as general informants. The sample was obtained by saturation of information. During data analysis, the four phases proposed by Leininger were applied: data collection, identification and categorization of descriptors and components, analysis of context and patterns and establishment of central themes. The results of this research provide theoretical knowledge to give culturally coherent care to pregnant women with preeclampsia and thus be a complement to their comprehensive care. Women require the health care team that serves them to inform them clearly and comprehensively about their health situation, that of their unborn child and the care they need. Women know about signs and alarming symptoms of preeclampsia, but do not perceive them. The emotional, social and spiritual changes produced in women by their illness and hospitalization need to be considered for nursing care.

Keywords: preeclampsia, pregnant women, qualitative research, nursing, transcultural nursing.

Key words plus: pre-eclampsia, pregnant women, qualitative research, nursing, transcultural nursing. 


\section{Significado que as grávidas internadas atribuem para a experiência de ter pré-eclâmpsia}

\section{Resumo}

Este estudo teve como objetivo descrever os significados que uma turma de mulheres grávidas internadas atribuiam à experiência de ter pré-eclâmpsia. Trata de pesquisa qualitativa tipo etnoenfermagem. Para a coleta da informação levou-se em conta a Guia observação-participação-reflexão, proposta por Leininger. Fizeram-se entrevistas abertas em profundidade a oito grávidas como informantes-chave e cinco enfermeiras como informantes gerais. A amostra foi obtida por saturação de informação. Na análise de dados foram aplicados os estágios propostos por Leininger: coleta da informação, identificação e categorização dos descritores e componentes, análise contextual e de padrões e estabelecimento de temas centrais. Os resultados da pesquisa aportam conhecimento teórico para brindar às grávidas com pré-eclâmpsia, cuido culturalmente congruente e, mesmo assim, ser complemento para seu atendimento integral. As mulheres precisam da equipe de saúde que serve para informá-las de maneira clara e abrangente sobre sua situação de saúde, a da criança por nascer e o cuido que elas estão precisando. As mulheres conhecem dos signos e sintomas de alarma da préeclâmpsia, mas elas não os percebem. As mudanças emocionais, sociais, espirituais produzidas na mulher, pela doença e internação precisam serem levadas em conta para o atendimento em enfermagem.

Palavras chave: pré-eclâmpsia, mulheres grávidas, pesquisa qualitativa, enfermagem, enfermagem transcultural.

Palavras chave descriptor: pré-eclâmpsia, gestantes, pesquisa qualitativa, enfermagem, enfermagem transcultural. 


\section{Introducción}

La preeclampsia representa un problema de salud pública, por ser una de las primeras causas de morbilidad y mortalidad materna y perinatal. Esta repercute no solamente en la mujer que padece la enfermedad, sino en el hijo por nacer, en la familia y en la sociedad $(1,2)$.

Sobre el tema de la preeclampsia se encuentran estudios e investigaciones con enfoque biomédico, de abordajes cuantitativos, con el fin de conocer aspectos fisiopatológicos de la enfermedad y tratamiento (3-6), pero son pocos los estudios que abordan la dimensión cultural de la gestante con diagnóstico de preeclampsia y sus experiencias ante esta situación de enfermedad, lo cual se requiere para generar propuestas de cuidado culturalmente congruentes a las necesidades de este tipo de población.

Según Strauss y Quinn, los significados son "la interpretación evocada en una persona por un objeto o evento en un tiempo determinado" (7). Según el Diccionario de la lengua española, la experiencia se define como el hecho de haber sentido, conocido o presenciado alguien algo, o circunstancia o acontecimiento vivido por una persona (8). En la investigación el significado de la experiencia se entendió como toda interpretación que tiene la mujer gestante acerca del hecho de tener preeclampsia.

El significado de las experiencias de los seres humanos en salud, enfermedad y muerte, como conocimiento fundamental para el cuidado de enfermería, debe estudiarse y analizarse desde la propia disciplina, no solamente en ámbitos comunitarios, sino en ámbitos hospitalarios, a fin de explorar el contexto cultural en el cual los seres humanos se desenvuelven. Por tal razón, este estudio pretendió conocer los significados que las gestantes hospitalizadas le atribuyen a la experiencia de tener preeclampsia y asî aportar un saber popular al saber científico para enriquecer las acciones del cuidado.

Para esta investigación, los significados desde lo cultural se abordaron desde el planteamiento de Leininger, quien toma los factores de la estructura social y cultural, constructo de la teoría de la universalidad y diversidad del cuidado cultural, que les dan significado a las expresiones, a las interpretaciones y a las interacciones sociales de las personas, en este caso de la mujer con preeclampsia.

Los factores de la estructura social y cultural (tecnológicos, religiosos y filosóficos, familiares y sociales, culturales, políticos y legales, económicos y educacionales) (9) están representados en el modelo del sol naciente, el cual permitió guiar a las investigadoras, pues proporciona un acercamiento verdaderamente holístico para conocer y entender a las gestantes con preeclampsia y así identificar los significados culturales de las mujeres del estudio con su situación de enfermedad (10).

Los hallazgos de esta investigación proporcionan conocimientos teóricos para que los profesionales de enfermería cumplan con requerimientos del cuidado y de las demandas sociales de la profesión, al asumir la importancia que se debe dar a elementos conceptuales como el significado, el cual forma parte de la práctica y la asistencia del cuidado de enfermería. 


\section{Metodología}

Se realizó una investigación cualitativa tipo etnoenfermería que permitió descubrir, documentar e interpretar los significados y las experiencias de salud-enfermedad en un grupo de gestantes con preeclampsia (9).

El método de investigación de la etnoenfermería fue diseñado explícitamente por Leininger (1985). Es un método cualitativo que utiliza modos y procesos naturalísticos, inductivos y de descubrimiento abierto con diversas estrategias, técnicas para documentar, describir, entender e interpretar los significados, experiencias, símbolos de las personas y otros aspectos relacionados que influyen en los fenómenos de enfermería actuales y potenciales (11).

La investigación se realizó en un hospital de cuarto nivel en el servicio de hospitalización de ginecobstetricia. En este servicio, las pacientes atendidas son afiliadas al régimen contributivo y en su mayoría están residenciadas en la ciudad de Bogotá y municipios cercanos.

Se tuvo en cuenta la Guía de observación-participación-reflexión propuesta por Leininger, en la que se invirtió tiempo observando para hacer conciencia de la situación y el contexto, antes de entrar a ser participantes activas.

La muestra se hizo por saturación teórica, es decir, hasta cuando hubo una redundancia de información obtenida y analizada por las investigadoras y verificada por las informantes. Los datos se lograron con ocho informantes clave hospitalizadas con diagnóstico de preeclampsia, seleccionadas por conveniencia, por creer que vivir la experiencia de la preeclampsia suministra la suficiente información para entender el fenómeno que se estudió en esta investigación.

Los criterios de inclusión fueron ser gestantes hospitalizadas con diagnóstico de preeclampsia, no tener patología sobreagregada y estar en capacidad mental para comprender y responder a la entrevista realizada. Se incluyeron cinco informantes generales (enfermeras del servicio de hospitalización de obstetricia con experiencia de cuidar gestantes con diagnóstico de preeclampsia). Esta es una recomendación que formula Leininger para ampliar la información y compartir visiones relevantes y puntos de vista culturales (11).

A cada una de las informantes clave se les realizaron dos entrevistas abiertas a profundidad, con una duración de aproximadamente 25 a 40 minutos. Se buscó el momento en que ellas se encontraban sin interferencias, como la visita médica, la aplicación de intervenciones, la visita de familiares, entre otros; además, se facilitó un ambiente cómodo, se utilizó un diálogo coloquial, se brindó confianza y tranquilidad, para que pudieran hablar libremente.

La entrevista se inició preguntando la edad, la escolaridad, el estado civil, la ocupación y la edad gestacional, y se continuó con la pregunta orientadora: ¿cómo ha sido su experiencia desde que sabe que tiene preeclampsia? A partir de las respuestas que daban las participantes seguían nuevas preguntas que intentaban profundizar y ampliar la información. Durante esta recolección seis de las informantes clave tuvieron parto, motivo por el cual se entrevistaron en el posparto. 
A las informantes generales se les realizó una entrevista grupal, con un tiempo de duración de 50 minutos. Las preguntas surgieron a partir de datos suministrados por ellas mismas, con el fin de ampliar y profundizar sobre los temas surgidos de las participantes clave; adicionalmente, se revisaron las historias clínicas de las gestantes para profundizar en el motivo de consulta y enfermedad actual, edad gestacional en el momento del diagnóstico, días de hospitalización, vía del parto y edad gestacional al momento del parto. Lo anterior permitió una mejor comprensión y análisis de la información.

En el análisis de los datos de la investigación se aplicaron las cuatro fases propuestas por Leininger (11): en la primera se recolectó la información, para la cual se tuvo en cuenta la observación del entorno y de las informantes; ello permitió acercarse y familiarizarse con el fenómeno de estudio, para posteriormente realizar la entrevista sin dejar de observar y ser participantes activos con una permanente reflexión de lo que se observaba y lo que se obtenía mediante los datos recolectados en la entrevista, las notas de campo y la revisión de historias clinicas. En la segunda fase se tomaron fragmentos de la información suministrada por las informantes clave (descriptor) y se les asignaron códigos (interpretaciones conceptuales del investigador al hacer un primer análisis). Por último, los códigos se agruparon por similitudes, teniendo en cuenta las notas de campo que contenian las observaciones realizadas y los factores de la estructura social y cultural del modelo del sol naciente.

En la tercera fase, los datos se estructuraron para descubrir la saturación de ideas y patrones recurrentes. También se examinaron para mostrar patrones con respecto a los significados en contexto. En esta fase también se contó con la información suministrada por las informantes generales, para tener mayor credibilidad y confirmación de los hallazgos. En la cuarta fase de análisis se identificaron los temas principales, hallazgos de la investigación teniendo en cuenta los factores de la estructura social y cultural del modelo del sol naciente y se plantearon algunas recomendaciones.

Los criterios de rigor considerados, de acuerdo con Leininger, fueron: credibilidad, confirmabilidad, significado en contexto, patrones recurrentes, saturación y transferibilidad (11). En los aspectos éticos se tuvo en cuenta lo descrito en la Resolución 008430 de 1993 del Ministerio de Salud (12) y las recomendaciones éticas para las investigaciones de la Facultad de Enfermería de la Universidad Nacional de Colombia (2008) (13). En primera instancia, el trabajo se presentó al Comité de Investigación y Ética de la Universidad Nacional de Colombia y del hospital donde se realizó el estudio. Además, se presentó de forma verbal y escrita el consentimiento informado a las informantes, a fin de darles la oportunidad de expresar inquietudes y dudas, haciendo las correspondientes aclaraciones, y se protegió la privacidad de las informantes escribiendo letras y números para su identificación, utilizando la siguiente nemotecnia: a cada informante clave y a cada entrevista se le asignó un número, por ejemplo, I1 corresponde a la informante 1 , de la entrevista 1. 


\section{Resultados}

La edad de las gestantes que participaron en la investigación oscilaba entre 21 y 33 años. El nivel de escolaridad fue bachillerato completo, tecnológico y universitario. Su estado civil estuvo entre casada, soltera y unión libre. Y la ocupación osciló entre ser ama de casa y estar empleada. La edad gestacional en la que les realizaron el diagnóstico de preeclampsia osciló entre la semana 29 y la 34, teniendo un tiempo de hospitalización aproximado de dos a ocho semanas. De las ocho informantes, a cinco se les realizó cesárea, una tuvo parto por vía vaginal y dos se encontraban en embarazo al finalizar la recolección de la información. De las seis informantes que tuvieron parto se pudo conocer que solamente una de ellas logró llegar a la semana 37 de gestación, y de las 5 restantes el parto fue entre la semana 32 y la 36. En cuanto a los recién nacidos, cuatro fueron hospitalizados en la unidad neonatal por ser pretérmino y dos fueron dados de alta junto con la madre.

A partir del análisis de la información surgen cuatro temas centrales con sus respectivos patrones recurrentes, en los que se refleja el significado que tiene para la gestante la experiencia de tener preeclampsia, es decir, las interpretaciones que hacen las gestantes sobre la experiencia de tener la enfermedad. A continuación se presenta los temas con cada uno de los patrones recurrentes hallados en la investigación.

\section{Tema 1. El cuidado de las gestantes teniendo en cuenta sus conocimientos}

El hecho de que las gestantes se encuentren en una situación que compromete su estado de salud y el de sus hijos por nacer es algo que no pasa inadvertido, razón por la cual adquieren información por parte del equipo de salud, familiares y factores tecnológicos como el internet (figura 1).

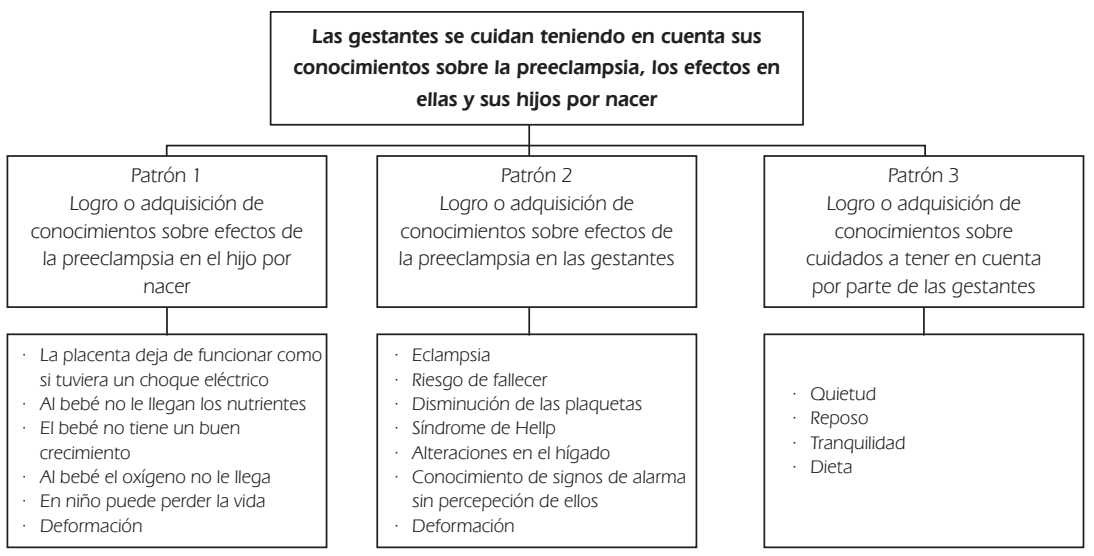

Figura 1. El cuidado de las gestantes teniendo en cuenta sus conocimientos FUENTE: elaboración propia. 
"Después de que yo vine acá (casa) me puse a mirar por internet para averiguar lo que no sabía" (I2E2). "Yo sé que al bebé puede que no le lleguen los nutrientes y hasta el niño pueda perder la vida... Al menos... eso fue lo que me dijeron en el otro hospital" (I2E1).

En cuanto al patrón 1 (adquisición de conocimientos sobre efectos de la preeclampsia en el hijo por nacer), la preeclampsia para ellas es conocida como alteración de la tensión arterial, algo peligroso, una enfermedad muy delicada que causa alteraciones en la placenta, como dejar de funcionar y como si tuviera un choque eléctrico; además, causa algunas complicaciones o repercusiones en su hijo por nacer, como que el bebé no reciba los nutrientes, no tenga un buen crecimiento, que el oxígeno no le llegue, que el bebé se asfixie y que pueda perder la vida:

La preeclampsia es tensión alta, que se le sube a uno la tensión y en este momento me imagino... No es que me lo hayan dicho sino que me imagino que como la placenta es prácticamente la energía del bebé, es lo que hace que el bebé esté bien, es como si tuviera un choque eléctrico, como si un cablecito en ese momento fallara. (I8 E2)

En el patrón 2 (adquisición de conocimientos sobre efectos de la preeclampsia en las gestantes), para algunas la preeclampsia es una alteración de la tensión arterial, algo peligroso que puede causar efectos en la salud de la madre y traer complicaciones como eclampsia, disminución de las plaquetas, síndrome de Hellp, afectación del hígado, y todo ello causar la muerte:

Cuando ya llega a ser eclampsia, ahí sí hay riesgo de que uno pueda fallecer, porque uno se inflama y aparte de que se inflama, pues que la tensión se sube y que se le pueden bajar las plaquetas y un poco de cosas más. (I2 E1)

En este patrón se pudo identificar que algunas de ellas conocen algunos signos y síntomas de la preeclampsia, como elevación de la tensión arterial, hinchazón, dolor de cabeza, visión borrosa, ver luces y escuchar pitos, aunque llama la atención que algunas informantes, a pesar de que refieren conocer los signos y síntomas de alarma, manifiestan que el motivo de consulta no se relaciona con estos o no era específico de la enfermedad: "me dijeron los sintomas de preeclampsia eran dolor en la boca del estómago, pitos en los oídos y ver lucecitas... Pero yo no tuve signos de preeclampsia" (I5 E1).

A pesar de que ellas informan que les explicaron y conocen los signos de alarma, estos son subvalorados y no reconocidos: "a mí nunca me dio nada", "no tuve signos de preeclampsia"; pero al momento de tener en cuenta la información registrada en la historia clínica, se identifica que ellas sí presentaron síntomas como edema y epigastralgia, los cuales no fueron reconocidos en el momento de presentarlos. 
Llama la atención que algunas gestantes informan que existe desinformación en relación con la enfermedad. En algunas ocasiones se identifica que no se les brinda información y, en otras, que no hay claridad:

Realmente un médico o alguien debería acercarse y decirle a uno "mire, Ud. tiene esto y esto; vamos a hacerle esto y esto", porque uno sabe es por lo que realmente uno pregunta o lo que a uno le dicen las enfermeras, al no darle a uno información eso genera incertidumbre. (I5 E2)

En cuanto a esto, la Resolución 13437 de 1991 (14), por la cual se constituyen los comités de ética hospitalaria y se adopta el Decálogo de los Derechos de los Pacientes, establece que todo paciente debe ejercer su derecho a disfrutar de una comunicación plena y clara con el médico, apropiada a sus condiciones psicológicas y culturales, que le permita obtener toda la información necesaria respecto a la enfermedad que padece, así como a los procedimientos y tratamientos que se le vayan a practicar y el pronóstico y riesgos que dicho tratamiento conlleve.

En el patrón 3 (adquisición de conocimientos sobre cuidados a tener en cuenta por parte de las gestantes) se identificó que el reposo o la quietud son cuidados que ellas conocen y contemplan. El no tener quietud lo relacionan con posible elevación de la tensión arterial y riesgo de complicaciones para la placenta y el bebé: "sí, prácticamente uno tiene que estarse quieta porque en cualquier cosa se le sube a uno la tensión y puede ser muy grave para la placenta y para el bebé" (I8 E2).

Por otro lado, refieren que la tranquilidad es un cuidado que deben tener. Para algunas de las informantes el estado de ánimo puede alterar la tensión arterial; consideran que la tranquilidad es un factor que beneficia la salud de la gestante con preeclampsia y, por ende, la salud del hijo por nacer: "sé que tengo que sentirme bien, que tengo que estar lo mejor posible, más tranquila, menos preocupada porque eso afecta la tensión y afecta todo" (I5 E1).

Para las informantes la dieta es considerada un cuidado, ya que esta puede favorecer al bebé. Al analizar los factores socioculturales del modelo del sol naciente, se puede identificar que el contexto de la hospitalización y el medio ambiente son favorables para cumplir con este cuidado, ya que ellas señalan que no es la misma alimentación que se consume en el hospital y la que se consume en la casa: "la alimentación no es lo mismo que tú comes acá a lo que comes en la casa, y creo que eso es lo que más puede cuidar al bebé" (I4 E1).

Teniendo en cuenta la estructura social y cultural del modelo del sol naciente, es posible identificar que la adquisición de conocimientos está influida por factores educacionales, dado que el nivel de escolaridad facilita comprender la información suministrada por parte del equipo de salud, aunque existe desinformación relacionada con falta de información por parte del equipo de salud. 
Factores tecnológicos como la monitoría fetal, el perfil biofísico, el examen Doppler fetoplacentario, la toma de tensión arterial y los exámenes paraclínicos son herramientas que permiten conocer el estado de salud de la gestante y de su hijo por nacer, lo que permite brindar y adquirir información sobre la enfermedad. Otro de los factores tecnológicos identificados es el internet.

Así mismo, existen factores políticos y legales que favorecen la información brindada, si se tiene en cuenta que el Código de Ética de Enfermería (15), en el capítulo III, establece que una de las responsabilidades de la enfermera con las personas que reciben sus servicios es dar información adecuada, suficiente y oportuna a la persona, a la familia y a la comunidad de acuerdo con sus necesidades, solicitud y capacidad de comprensión.

\section{Tema 2. Las gestantes vivencian repercusiones por la preeclampsia}

En cuanto al patrón 1 (reacción materna), señalado en la figura 2, las informantes, al conocer la presencia de enfermedad, piensan que la preeclampsia es una enfermedad sorpresiva e inesperada, ya que consideran ser personas sanas. La hospitalización les produce cambios en el estado de ánimo, como estar irascible e irritable, alteraciones en el sueño y el descanso y, unido a esto, la imposibilidad de dormir por factores contextuales y ambientales, como el ruido y el alojamiento en habitación compartida.

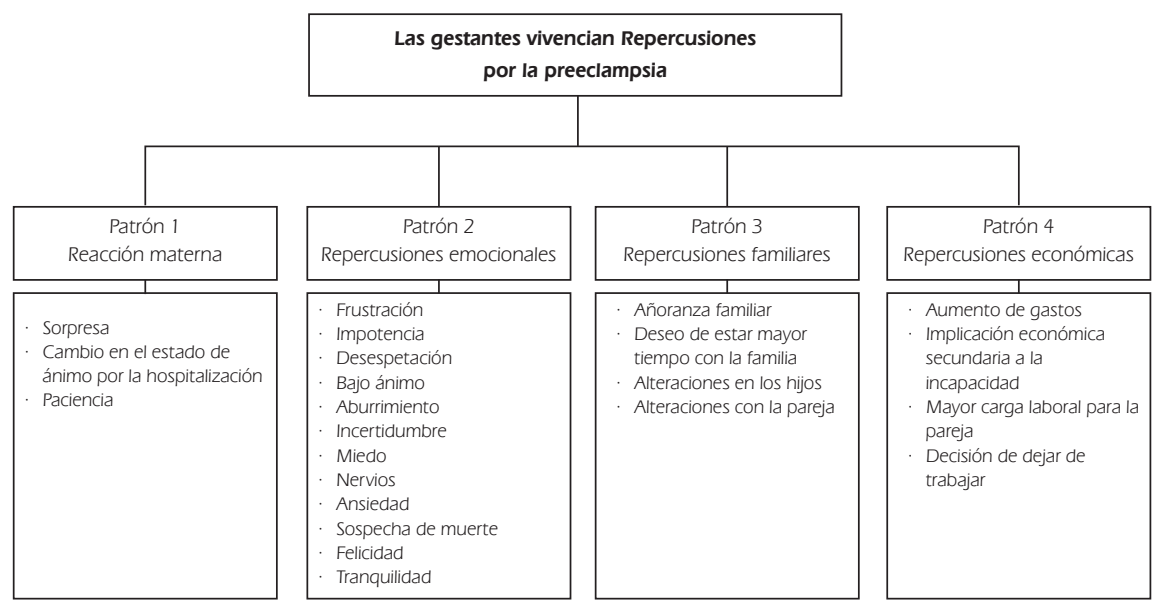

FiguRA 2. Las gestantes vivencian repercusiones de la preeclampsia FUENTE: elaboración propia. 
Al comienzo fue duro, pues yo no me lo esperaba, yo creía que mi embarazo iba a ser normal. (I6 E2)

A mí me afecta mucho el no dormir y allá era imposible dormir porque pues el ruido, adicionalmente que tiene una compañera uno al lado, que tenian de pronto un bebé ahí entonces pues uno no podía descansar [...] me controlaban las cifras de tensión cada hora cada dos horas era imposible poder conciliar el sueño por más de tres horas. (I2 E2)

Por el contrario, algunas de ellas deciden estar calmadas, no estresarse, tener paciencia y resignación frente a la hospitalización, reacciones que pueden generar mejor afrontamiento de la enfermedad:

Cuando me hospitalizaron yo me resigné a que tenía que estar acá, que tenía que estar calmada y tener paciencia, entonces pues mi decisión fue no estresarme y quedarme ya acá esperando que naciera mi bebé. (I6 E1).

En el patrón 2 (repercusiones emocionales) se identificó que la preeclampsia genera en la gestante hospitalizada sentimientos como incertidumbre, miedo, nervios y ansiedad, secundario a no saber qué va a pasar con su salud y con la salud del bebé por nacer, debido al desconocimiento de lo que sucederá a futuro y lo inesperado durante el trascurso de la enfermedad:

Tenía incertidumbre, porque... Yo no sabía qué iba a pasar, yo no sabía si la cesárea iba a salir bien o iba a salir mal, si en el transcurso a que cumpliera las 34 semanas iba a tener otra subida de tensión e iba a convulsionar y no sabía qué le iba a pasar a mi bebé y a mí. (I5 E2)

Cuando las gestantes conocen que tienen preeclampsia, presentan sentimientos relacionados con la muerte de sí mismas y de sus hijos por nacer; piensan que se van a morir y buscan planear el futuro de sus hijos:

Incluso yo llegué a decirle a mi esposo que si yo me llegaba morir, que pues cuidara a los niños [...] yo a toda la familia le decía que por favor me cuidaran los niños, porque yo pensaba era que muy seguramente a mí algo me iba a pasar [...] cuando me hospitalizaron uno firma un consentimiento pues no le dicen eso, pero dice allí que uno puede morir en el parto. (I2 E2)

Otra de las repercusiones emocionales identificadas es tranquilidad frente a la hospitalización, a la atención recibida y a la información suministrada por el equipo de salud: "Después de la monitoría se tranquiliza uno porque los doctores dicen que todo está bien” (I3 E1).

En el patrón 3 (repercusiones familiares) se conoció que durante la hospitalización se presenta añoranza familiar, dado que extrañan y les hacen falta sus hijos, el esposo y el amor de la familia. Tal situación las afecta anímicamente y en ellas genera aburrimiento: 
Anímicamente ya me he tratado de deprimir aquí encerrada, pues porque me hace falta mi familia, a pesar de la comodidad que se siente acá no es lo mismo que estar uno en su casa con su familia y disfrutando con ellos. (I1 E1)

El hecho de tener la posibilidad de estar con un acompañante por la noche hubiera sido fabuloso para mí, o sea alguien que estuviera, o que le dieran la posibilidad a mi hijo de entrar, porque yo sé que es un hospital y todo pero él es un niño de cinco años y yo le hago falta. (I2 E1)

Como consecuencia de la situación de enfermedad de la gestante y la necesidad de hospitalización, en los hijos que están en casa surgen una serie de reacciones, referidas por ellas como mal comportamiento, bajo rendimiento académico, rebeldía, llanto y depresión, y en la pareja, mayor carga económica, carga laboral e implicación en la vida sexual:

Ya se empiezan a ver las consecuencias: mi hijo, a pesar de su edad, no entiende mucho lo que está pasando, pero él lo demuestra con su comportamiento, entonces se ha empezado a portar mal, ha bajado su rendimiento académico. (I1 E1)

Mi esposo tuvo una carga económica más que asumir y el hecho de que mi esposo no pudiera estar conmigo a toda hora, ya que él tenía que trabajar.

Yo tendría seis meses alguito, casi siete y pues no pude volver a tener relaciones con mi esposo. (I2 E2)

En el patrón 4 (repercusiones económicas) se conocieron algunas implicaciones como aumento de gastos secundario a la prematurez y hospitalización del recién nacido, necesidad de incapacidad médica, aumento de la carga laboral de la pareja para asumir los gastos del hogar, lo cual condiciona tener menor tiempo para compartir con la gestante y, para una de ellas, tener que dejar de trabajar para cuidarse durante el embarazo:

Más tiempo acá en la unidad y eso significa también que el bolsillo nos toca más duro, porque igual siempre han sido gastos fuertísimos, los solos pañales de prematuro son carísimos, más caros que los pañales para un bebé normal. (I1 E2)

Prácticamente la preeclampsia sí me ha afectado, porque obviamente no no estoy produciendo, pero de todas formas pues toca esperar a ver qué pasa, pero de todas formas como yo estaba trabajando pues igualmente el sueldo me irá a llegar de acuerdo a la incapacidad. (I8 E2)

Yo llevo como mes y medio sin trabajar y la EPS no me quiere pagar la incapacidad de los cuarenta dias porque la de los ochenta y algo yo me imagino que me la tendrán que pagar, pero me han puesto mucho problema con lo de las incapacidades. (I7 E2)

Teniendo en cuenta lo expresado por las gestantes, se identifica que la familia y factores sociales hacen parte de la reacción materna, ya que 
ellas presentan añoranza familiar y desean o anhelan estar con su familia. Del mismo modo, factores ambientales de la hospitalización conducen a alteraciones en ellas que están relacionadas con la falta de descanso, cambios en el estado de ánimo, sentimientos de ansiedad y aburrimiento, aunque cabe resaltar que el hecho de estar hospitalizadas genera tranquilidad al percibir control de la enfermedad y tratamiento especializado.

También se identifican alteraciones en los factores económicos, ya que la hospitalización genera gastos adicionales que repercuten en la economía familiar. De igual forma, los factores politicos y legales influyen en la gestante, si se piensa que en cuanto a las incapacidades, los derechos de algunas gestantes están siendo vulnerados de acuerdo con lo estipulado por la Ley 100 de 1993, en el artículo 206 (16).

\section{Tema 3. Las gestantes valoran su situación de enfermedad desde diferentes contextos}

En el patrón 1 (valoración que hacen las gestantes de sí mismas), señalado en la figura 3, se identifica que antes de realizarles el diagnóstico de preeclampsia y después de realizado, ellas no aceptan la enfermedad, porque se sienten bien y no perciben sintomas de alteración. Lo anterior llama la atención, dado que las gestantes, al no sentirse enfermas, no consultan oportunamente y cuando lo hacen, ya pueden existir alteraciones en la salud del binomio madre-hijo; adicionalmente, dicha percepción puede causar no adherencia en la hospitalización y manejo, ya que no ven la necesidad de ser controladas y tratadas: "pues yo aparentemente me siento bien, entonces por qué no me puedo ir si yo me siento ya mejor” (I2 E1).

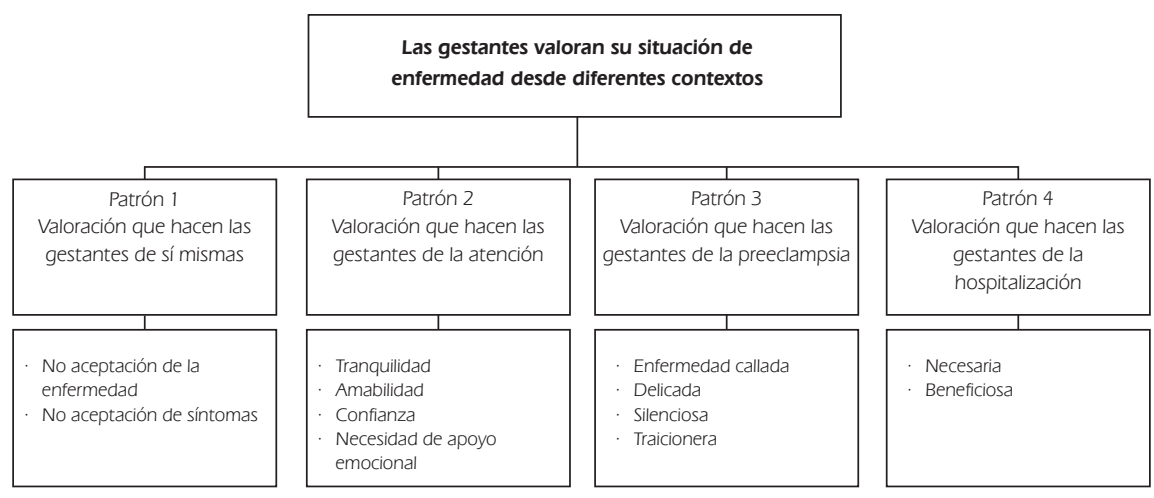

FiguRA 3. Las gestantes valoran su situación de enfermedad desde diferentes contextos FUENTE: elaboración propia. 
En cuanto al patrón 2 (valoración que hacen las gestantes de la atención del equipo de salud) refieren sentir tranquilidad, ya que se sienten controladas y perciben que de esta forma su salud y la de su hijo no corre riesgo; además, dicen que la atención es amable y que el equipo de salud está pendiente de su situación de enfermedad, lo cual genera confianza frente a la atención recibida: "me controlan y vienen controlando esa enfermedad... Hay más probabilidades de que mi vida no corra riesgo y que la de mi bebé tampoco, eso es positivo, es bueno..." (I1 E1).

Además, consideran que las intervenciones de enfermería están orientadas a la realización de actividades técnico-asistenciales enfocadas en la dimensión fisiopatológica de la enfermedad, pero no en la dimensión emocional. Al respecto justifican que la carga laboral de las enfermeras es alta, motivo por el cual no pueden brindar cuidado integral a la gestante:

Las enfermeras tratan de que uno esté cómodo, de que uno esté bien, de que cualquier cosa que uno sienta ellas puedan solucionar, pero en cuanto a lo anímico ellas no se dan mucha cuenta de cuando uno está aburrido. (I5 E1)

Uno entiende que las enfermeras tienen muchas más pacientes y muchas más cosas que hacer, que ponerse a anímicamente estabilizar la gente... (I1 E1)

En el patrón 3 (valoración que hacen las gestantes de la preeclampsia), ellas expresan que es una enfermedad "callada", "delicada" y "silenciosa", dado que en muchas ocasiones ellas no experimentan signos y sintomas; además, mencionan que es una enfermedad traicionera, pues en el momento menos esperado puede causar alteraciones en la madre o el hijo:

Uno como tal no siente nada, es una enfermedad como muy callada, uno no siente nada, solo en el momento que uno tenga una tensión alta puede sospechar de ella, pero puede traer bastantes complicaciones como me pasó a mí. (I4 E2)

Pienso que la preeclampsia es una enfermedad delicada y silenciosa; me di cuenta mientras estuve hospitalizada que es una enfermedad que traiciona en cualquier momento. (I6 E2)

En el patrón 4 (valoración que hacen las gestantes de la hospitalización) se pudo identificar que, a pesar de que desean estar con su familia, la hospitalización es vista como algo necesario y beneficioso, motivo por el cual se resignan y la aceptan:

Yo creo que lo mejor que uno puede hacer es estar en la casa con su familia, pero por el bien del bebé toca quedarse aquí. (I3 E1)

Yo sabía que en el momento que estuviera acá en el hospital iba a estar bien y que en cualquier momento me podian desembarazar. (I4 E2) 
Según los factores de la estructura social y cultural, se puede apreciar que ellas valoran que las intervenciones del equipo de salud están influenciadas por factores tecnológicos que les permiten brindar un adecuado cuidado orientado a la identificación del diagnóstico y tratamiento. E1 contexto y el medio ambiente de la hospitalización permiten que el equipo de enfermería y las gestantes tengan una relación de empatía, que logra redirigir el cuidado técnico-asistencial y brindar intervenciones que favorezcan emocionalmente a las pacientes.

En la valoración que las gestantes hacen de la preeclampsia mencionan que es una enfermedad traicionera que puede causar alteraciones en la madre y el hijo, lo cual tiene influencia en los factores sociales y familiares, si se tiene en cuenta que la preeclampsia puede causar múltiples implicaciones en la familia y en la sociedad.

\section{Tema 4. Las gestantes toman nuevas decisiones en sus vidas va causa de la preeclampsia}

En el patrón 1 (decisiones relacionadas con la utilización del tiempo), visto en la figura 4, ellas manifiestan que durante la hospitalización realizan pocas actividades de distracción o entretenimiento, las cuales se ven limitadas por el ambiente de la hospitalización: "no es mucho lo que se puede caminar acá" y por su estado de salud "ahora ya ni me puedo sostener en pie porque me da como ahogo"; situaciones que genera en ellas aburrimiento e inconformidad: "llega un punto en el que uno no sabe ni qué hacer":

Ver televisión, pues cuando podía visitarme mi esposo pues hablar con ellos, la compañía es supremamente importante y leer un libro, acá tengo libros, escuchar música. Ya llega un punto en el que uno no sabe ni qué hacer. (I2 E1)

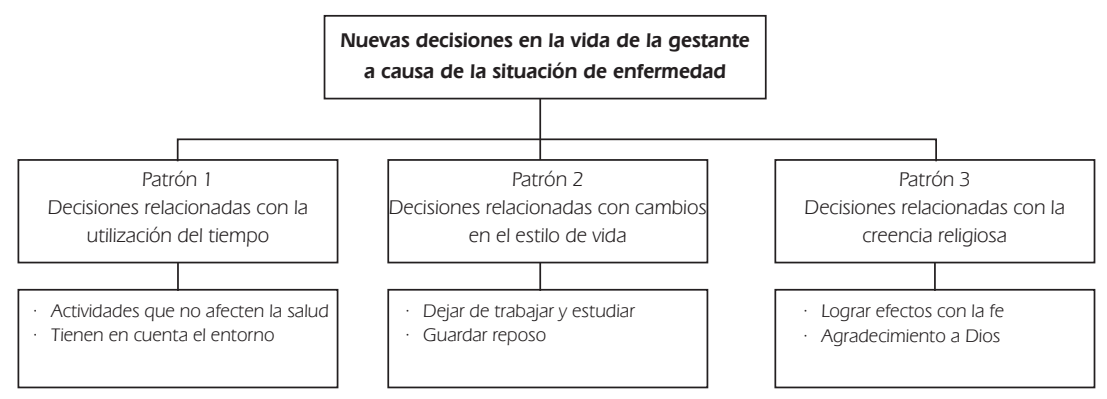

FiguRA 4. Las gestantes toman nuevas decisiones en sus vidas a causa de la preeclampsia FUENTE: elaboración propia. 
En el patrón 2 (decisiones relacionadas con cambios en el modo de vida) se conoció que ellas identifican que la presencia de enfermedad condiciona un cambio en el modo de vida, bien sea por voluntad propia o porque la presencia de enfermedad lo ha generado; asî es como algunas han tenido que dejar de trabajar y de estudiar:

La enfermedad a uno lo reduce, después de que uno se mueve y que lo reduzcan a uno a tanto es complicado, no puedo moverme, no me puedo parar así mucho, no puedo agitarme, no puedo tener nada porque tengo que estar con mi tensión normal para que él bebé no tenga ningún problema. (I8 E1)

En el patrón 3 (decisiones relacionadas con la creencia religiosa) se conoció que cuando a la gestante le diagnostican preeclampsia y sabe el riesgo que implica la enfermedad para su salud y la de su hijo por nacer, ellas se aferran a creencias religiosas que le permiten tener un mejor afrontamiento de la enfermedad. Tales creencias están orientadas a pedirle a Dios para lograr bienestar en su salud, en la del hijo por nacer y dar agradecimiento a Dios por los favores:

Cuando rezo siento más tranquilidad, que le estoy pidiendo a mi Diosito que salve la vida de mi hija, que todo salga bien en el parto. (I1 E1)

Me daba miedo pero me aferraba mucho en Dios, cada vez me aferraba más a Dios y ponía en las manos de Dios mi salud y la de mi bebé, y pues acá estoy bien, feliz y contenta. (I6 E2)

Gracias a Dios yo por lo menos alcancé a la semana 34, porque me di cuenta que hay mujeres que llegan de semanas 27 y 28 y de una vez las tienen que desembarazar y se les complica todo, se complican los bebés, porque no crecen, le doy gracias a Dios que mi bebé alcanzó a crecer. (I6 E2)

De acuerdo con lo planteado por Leininger en el modelo del sol naciente, se puede apreciar que la utilización del tiempo y los modos de vida están influenciados por factores como el contexto y el medio ambiente, ya que las actividades de distracción o entretenimiento se ven limitadas por el ambiente de hospitalización en el que se encuentra, además el hecho de que la mujer esté hospitalizada implica cambios en su vida como dejar de trabajar y estudiar y limita la realización de las actividades diarias, no solo por estar hospitalizada, sino por su condición de enfermedad.

En este patrón también se puede identificar que las creencias religiosas están relacionadas con factores familiares, ya que estas son trasmitidas de generación en generación, tienen arraigo cultural y están influenciadas por la familia; además, se relacionan con el contexto, el medio ambiente de la hospitalización y la situación de enfermedad, factores propicios para que la gestante realice o practique creencias religiosas para su bienestar. 


\section{Discusión}

De acuerdo con los significados que las gestantes hospitalizadas le atribuyen a la experiencia de tener preeclampsia, en el tema 1 (el cuidado de las gestantes), según sus conocimientos, se identificó que, a pesar de que ellas informan que les explicaron y conocen los signos de alarma, estos son subvalorados y no reconocidos; de igual forma, la investigación realizada por Pérez Belia (17) sobre el significado que las puérperas asignan a la experiencia de una gestación con preeclampsia reporta que las puérperas del estudio consideran el "embarazo como un proceso normal", de tal manera que los signos y síntomas que presentan cuando tienen la preeclampsia, son percibidos por ellas como normales y como parte del proceso natural de la gestación, y tal situación puede limitar la atención oportuna de la gestante.

En esta investigación, las gestantes con preeclampsia guardan reposo o quietud para cuidar su salud y la de su hijo por nacer. Contrario a lo que se esperaba, no se encontró evidencia de dicho cuidado. Al respecto, una revisión del tema hecha por Cochrane, en el 2008 (18), reportó que, a la fecha, las pruebas para apoyar la recomendación del reposo o de la actividad reducida para prevenir la preeclampsia y sus complicaciones eran insuficientes.

En relación con el significado que las gestantes le atribuyen a las repercusiones que vivencian a causa de la preeclampsia, se coincide con Medina Cordero y colaboradores (19), respecto a que la mayoría de las pacientes no descansan ni duermen bien durante su estancia en el hospital, unas veces por el proceso patológico que están viviendo y en otras ocasiones debido a causas ambientales como ruido, iluminación, falta de intimidad e interrupciones por procedimientos terapéuticos, por lo cual el patrón normal del sueño puede verse alterado, lo que constituye un factor negativo que repercute e influye en el estado del paciente.

Así mismo, se coincide con Pinho Carvalheira (20), Acevedo (21) y Pérez Belia (17), al encontrar que, ante la presencia de enfermedad, se manifiestan sentimientos relacionados con la muerte. La muerte de una madre es una tragedia evitable que afecta una vida joven llena de grandes esperanzas; es una de las experiencias más traumáticas que puede sufrir una familia y afecta seriamente el bienestar, la supervivencia y el desarrollo de los hijos, especialmente de los más pequeños (22).

Una de las interpretaciones que hacen las gestantes a causa de su enfermedad es la autovaloración, lo cual coincide con lo identificado en la investigación sobre el significado que las puérperas asignan a la experiencia de una gestación con preeclampsia realizada por Pérez Belia (17), al encontrar que son ellas las que se autovaloran y, así, se alejan de la posibilidad de detectar signos y sintomas de alarma, que conduzcan a hacer un diagnóstico precoz y tratamiento oportuno, y sobre todo evitar las consecuencias de deterioro de la preeclampsia, en la salud de las madres y sus hijos por nacer.

Las gestantes, a partir de la experiencia de tener preeclampsia, consideran que las intervenciones de enfermería están orientadas a la realización de actividades técnico-asistenciales, pero no a la dimensión emocional; 
contrario a lo que se espera, sí se aplica la Guía de atención de las complicaciones hipertensivas asociadas con el embarazo (23), en la cual se recomienda que el equipo de salud brinde a la madre, a su pareja y a su familia, además de atención profesional eficiente, apoyo psicológico, consejería y trato amable, prudente y respetuoso, que les permita tener tranquilidad y seguridad durante el tratamiento de la complicación hipertensiva.

A pesar de que el equipo de enfermería brinda cuidados acordes con la alteración de salud de la gestante, se debe tener en cuenta no solamente el cuidado técnico asistencial, sino también el componente psicosocial, sus necesidades de cuidado y su cultura, a fin de brindar un cuidado de enfermería culturalmente congruente, holístico y humanizado. Desde esta misma perspectiva, Ibarra y Gonzales (24) señalan que el compromiso social que tiene la enfermera para cuidar a la persona, a la familia y a la comunidad, requiere que, además del conocimiento de la técnica, de los aspectos instrumentales del cuidado y de la comprensión intelectual, exista el conocimiento de esa otra persona, por medio de una relación holística. La actual dinámica social hace que el cuidado de enfermeria se convierta en un desafio permanente, pues debe considerar los valores, creencias y prácticas de individuos que tiene bajo su cuidado, así como manifestar el respeto por la diferencia cuando ofrece dicho cuidado.

La experiencia de tener preeclampsia para las gestantes significa tomar nuevas decisiones y hacer cambios en los modos de vida, lo cual coincide con Gallo y colaboradores (25), al señalar que el individuo hospitalizado se ve limitado en su acción por el problema de salud que lo afecta, el medio que lo rodea y el personal de salud que lo atiende. En este contexto, la actividad física, recreativa y de relajación se convierte en una herramienta terapéutica que permite obtener bienestar físico; proporcionar la oportunidad de aprender, crear, divertirse, y establecer relaciones interpersonales.

En cuanto a las decisiones relacionadas con la creencia religiosa, Pérez Belia (17) identificó que la fe en un ser superior, en Dios, es algo que también fortalece y les ayuda a estar optimistas y estar esperanzadas. Después de que reconocen el trastorno y saben de las alteraciones que su hijo puede tener, ellas fortalecen su vínculo con el ser supremo, y confian en que Él permitirá tener un hijo sano. Del mismo modo, Sánchez Herrera menciona que la dimensión espiritual humana es esencial para alcanzar un nivel óptimo de salud, de bienestar o de calidad de vida. Lo espiritual se desarrolla en cada persona a través de la espiritualidad, como una cualidad suprema que da significado. Cada ser humano requiere cuidado espiritual que le permita crecer como persona que satisface sus necesidades espirituales, y la religión puede ser un medio o una forma de vida que apoye el cumplimiento de dichas necesidades (26).

\section{Conclusiones}

Las gestantes hospitalizadas, a partir de la experiencia de tener preeclamp44 sia, se cuidan según los conocimientos que tienen en relación con la enfermedad y los efectos que esta pueda tener en ellas y sus hijos por nacer. 
La experiencia de tener preeclampsia genera en las gestantes sentimientos de incertidumbre, ansiedad y miedo, especialmente a la muerte materna; además, con la hospitalización surgen cambios en el modo de vida, al tener que alejarse de su familia o dejar de trabajar y de estudiar por guardar reposo. En algunos casos ven disminuidos sus ingresos económicos, se altera su patrón normal de sueño, se produce aburrimiento, preocupación y pocas opciones para utilizar el tiempo.

La gestante le asigna diferentes significados a la situación de enfermedad, esto si se tiene en cuenta la valoración que hace de sí misma, de la atención recibida, de la enfermedad y de la hospitalización; además, identifica la no aceptación de la enfermedad secundaria a la falta de precepción de los sintomas, sentimientos de tranquilidad, confianza y necesidad de apoyo frente a la atención recibida, además de concebir la hospitalización como necesaria y beneficiosa, si se tiene en cuenta que para ellas la preeclampsia es una enfermedad callada, delicada, silenciosa y traicionera.

Como significado de la experiencia de tener preeclampsia, durante la enfermedad las mujeres del estudio tienen más apego por las creencias religiosas, pues la situación acrecienta la fe en Dios y genera agradecimiento por los favores recibidos.

\section{Recomendaciones}

Durante el control prenatal de la gestante, el profesional de enfermería debe incluir conocimientos generados por investigaciones como esta, que muestran la necesidad de indagar en las mujeres la identificación de signos y sintomas de alarma para la preeclampsia, no solamente desde su conocimiento, sino también desde su percepción.

En el cuidado de la gestante hospitalizada con preeclampsia, identificar las necesidades de cuidado, trascendiendo de la dimensión fisiopatológica, hacia las dimensiones social, familiar, cultural, emocional y espiritual.

La enseñanza de enfermería para el cuidado de la mujer gestante con preeclampsia debe estar orientada hacia el conocimiento que produce la disciplina de enfermería, a fin de trascender lo biomédico y enfocarse en lo que las mujeres experimentan y requieren para ser cuidadas de manera integral.

Se proponen investigaciones en las cuales se avalúen intervenciones de cuidado que surjan de las necesidades de cuidado expresadas por las mujeres y valoradas por el profesional de enfermería durante el proceso de su enfermedad con preeclampsia.

\section{Agradecimientos}

Las autoras agradecen a la Pontificia Universidad Javeriana y a las informantes clave y generales del estudio. Este trabajo estuvo enmarcado en los proyectos del grupo de investigación en cuidado materno-perinatal de la Universidad Nacional de Colombia y contó con el apoyo financiero de la División de Investigación Bogotá (DIB) de la Universidad Nacional de Colombia. 


\section{Referencias}

1. Sáez Cantero V, Pérez Hernández MT, Aguero Alfonso G, González García H, Alfonso Dávila A. Resultados perinatales relacionados con los trastornos hipertensivos del embarazo. Rev Cubana Obstet Ginecol. 2012;38(1):36-44.

2. Díaz LA, Serrano NC. Oportunidades de investigación en preeclampsia, desde la perspectiva de prevención primaria. Rev Colomb Obstet Ginecol. 2008;59(3):206-15.

3. Suárez González JA, Cabrera Delgado MR, Gutiérrez Machado M, Corrales Gutiérrez A, Cairo González V, Rodríguez Royelo L. Resultados de la atención a pacientes con riesgo de preeclampsia-eclampsia. Rev Cubana Obstet Ginecol. 2012;38(3):305-12.

4. Serrano Díaz N C, Páez Leal M C, Beltrán Avendaño M A, Colmenares Mejía CC, Guio Mahecha E, Bautista Niño P. Preeclampsia y riesgo cardiovascular: estudio de seguimiento en la población de GenPE en Colombia. Rev Colomb Obstet Ginecol [internet]. 2012 [citado 2013 ago 10];63(3). Disponible en: http://www.fecolsog.org/userfiles/file/ revista/Revista_Vo163No3_Julio_Septiembre_2012/v63n3a07.htm.

5. López Jaramillo P, Silva F, Camacho PA, Pradilla LP, García R, Rueda Clausen C et al. Síndrome metabólico y preeclampsia: los aportes realizados por el Instituto de Investigaciones de la Fundación Cardiovascular de Colombia. Rev Col Cardiol [internet]. 2006 [citado 2010 ago 10];13(2). Disponible en: http://www.scielo.org.co/scielo. php?script $=$ sci_arttext\&pid $=$ S0120-56332006000500006\&lng=en .

6. Domínguez Anaya R, Herazo Beltrán Y. Edad de la gestante adolescente como factor de riesgo párrafo complicaciones en el embarazo. Cartagena Colombia 2009: estudio de corte transversal. Rev Colomb Obstet Ginecol [internet]. 2011 [citado 2010 ago 10];62(2). Disponible en: http://www.scielo.org.co/scielo.php?script=sci_ arttext\&pid=S0034-74342011000200004\&lng=en.

7. Strauss C. Quinn N. A cognitive theory of cultural meaning. Cambridge: Cambridge University Press; 1997.

8. Real Academia Española. Diccionario de la lengua española: experiencia [internet]. Disponible en: http://lema.rae.es/ drae/?val=experiencia.

9. Leno D. Buscando un modelo de cuidados de enfermería para un entorno multicultural. Gaceta de Antropología [internet], 2006 [citado 2011 ene 16];32(22):115-43. Disponible en: http://www.ugr. es / pwlac/G22_32Daniel_Leno_Gonzalez.pdf.

10. Leininger M. Culture care assessment to guide nursing practices. In: Transcultural nursing concepts, theories, research $\&$ practices. 2nd ed. New York: McGraw Hill; 1995.

11. Leininger M, McFarland M. Culture care diversity and universality: a worldwide nursing theory. 2nd ed. Sudbury, Massachusetts: Jones and Bartlett Publishers; 2006. 
12. Resolución 008430/1993 de 4 de octubre, por la cual se establecen las normas científicas, técnicas y administrativas para la investigación en salud. República de Colombia, Ministerio de Salud [internet]. Disponible en: http://www.urosario.edu.co/urosario_files/a2/ a24fb07a-f561-4fcc-b611-affff4374bb7.pdf.

13. Universidad Nacional de Colombia, Facultad de Enfermería, Equipo Comité de Ética. Recomendaciones éticas para las investigaciones en la Facultad de Enfermería de la Universidad Nacional de Colombia. Bogotá; 2008.

14. Resolución 13437/1991 del 1 de noviembre, por la cual se constituyen los comités de ética hospitalaria y se adopta el decálogo de los derechos de los pacientes. República de Colombia, Ministerio de Salud [internet]. Disponible en: http://www.ipsuniversitaria.com. co/documentos/Comite_de_etica_hospitalaria/Resolucion_13437_ de_1991.pdf.

15. Tribunal Nacional Ético de Enfermería. Código de Ética de Enfermería. Santa Fe de Bogotá, Colombia. 1998 [internet]. [Consultado 2012 mar 30]. Disponible en: http://www.trienfer.org.co/index. php?option $=$ com_content\&view $=$ article $\& i d=49 \&$ Itemid $=63$.

16. Ley 100/1993. Artículo 206 [internet]. Disponible en: http://www. secretariasenado.gov.co/senado/basedoc/ley/1993/ley_0100_1993_ pr006.html.

17. Pérez Herrera B. Significado que las puérperas asignan a la experiencia de una gestación con preeclampsia [tesis de Maestría en internet]. Bogotá: Universidad Nacional de Colombia, Facultad de Enfermería; 2011 [consulta 2012 mar 30]. Disponible en: http://www. bdigital.unal.edu.co/5415/.

18. Meher DL. Reposo durante el embarazo para la prevención de la preeclampsia y sus complicaciones en mujeres con presión arterial normal (Revisión Cochrane traducida) [internet]. En: La Biblioteca Cochrane Plus. Oxford: Update Software ; 2008 [consulta: 2012 mar 15]. Disponible en: http://www.update-software.com.

19. Medina Cordero A, Feria Lorenzo D, Oscoz Muñoz G. Los conocimientos sobre el sueño y los cuidados enfermeros para un buen descanso. Revista Enferm Glob [internet]. 2009 [citado 2012 abr];17. Disponible en: http://scielo.isciii.es/scielo.php?script=sci_ arttext\&pid=S1695-61412009000300005\&lng=es.

20. Pinho Carvalheira AP, Pamplona Tonete L, García C. Sentimientos y percepciones de mujeres en el ciclo embarazo-puerperio que sobrevivieron a una morbosidad materna grave. Rev Latino-Am Enfermagem [internet]. 2010;18(6). Disponible en: http://www.scielo.br/ pdf/rlae/v18n6/20.pdf.

21. Acevedo D, Pinherio D, Costa I, Medeiros A. Percepciones y sentimientos de mujeres gestantes y puérperas sobre la preeclampsia. Rev Salud Pública [internet]. 2009 [citado 2012 oct];11(3). Dispo- 
nible en: http://www.scielosp.org/scielo.php?script=sci_arttext\&pi $\mathrm{d}=\mathrm{S} 0124-00642009000300004$.

22. Secretaría de Salud de Medellín. Modelos para el análisis de la mortalidad materna y perinatal. Medellin; 2005. Disponible en: http:// www.nacer.udea.edu.co/libros.htm.

23. Ministerio de la Protección Social de Colombia. Guía de atención de las complicaciones hipertensivas asociadas con el embarazo. Bogotá: autor; s. f.

24. Ibarra Mendoza TX, González J. Competencia cultural: una forma humanizada de ofrecer cuidados de enfermería. Index Enferm [internet]. 2006 [citado 2012 oct];15(55). Disponible en: http://scielo.isciii.es / scielo.php?script=sci_arttext\&pid=S1 132-12962006000300010\&lng=es. doi: $10.4321 / \mathrm{S} 1132-12962006000300010$.

25. Gallo Sáenz D, Vélez Rúa D, Correa Arroyave M, Ortiz M. Utilización del tiempo libre en pacientes hospitalizados. Invest Educ Enferm. 1993;11(1):35-46.

26. Sánchez Herrera CB. Dimensión espiritual del cuidado de enfermería en situaciones de cronicidad y muerte [documento de trabajo en internet]. Bogotá: Universidad Nacional de Colombia; 2004. Disponible en: http://www.bdigital.unal.edu.co/2050/. 